Supramolecular chemistry

\title{
Molecular merry-go-round
}

The ingenuity of chemists has produced molecules that mimic a range of mechanical devices, from rotors and shuttles to switches, turnstiles and ratchets. Hiraoka, Shiro and Shionoya have now created a molecular ball-bearing, an assemblage of two disc-shaped molecules that rotate relative to each other on silver ions $\left(\mathrm{Ag}^{+}\right)$sandwiched between them (J. Am. Chem. Soc. 126, 1214-1218; 2004).

As shown in the figure, the discshaped molecules consist of a central benzene ring carrying either alternating thiazolyl and $p$-tolyl rings (top) or six thiazolyl rings (bottom). Mixing solutions of these molecules with silver ions results in the spontaneous formation of the ballbearing complex. This is most stable when each silver ion is linearly coordinated to two nitrogen atoms, one each from a thiazolyl group in the upper and lower molecules. Trigonal coordination is also possible, between a silver ion and two neighbouring thiazolyl nitrogen atoms in the lower molecule and one thiazolyl nitrogen atom in the upper molecule. The complex can shift from linear coordination to trigonal to linear again, as each silver ion exchanges its original partner on the lower molecule for a nitrogen atom on a neighbouring thiazolyl group of the same molecule. During each exchange, the two discs rotate through $60^{\circ}$ relative to each other. Hiraoka et al. use NMR spectroscopy to watch the ballbearing in action. Close to room temperature, the complex is in full swing, like a spinning carousel, and the coordination exchanges are faster than the timescale of NMR response. But if the temperature is lowered, the coordination exchange and hence the rate of rotation, slows down. Then the NMR data clearly reveal that the six thiazolyl groups of the lower molecule experience two distinct chemical environments, with half of the groups coordinated to silver ions whereas the other half remain uncoordinated.

The task now is to control the speed and direction of the rotation - and put the device to practical use. Magdalena Helmer
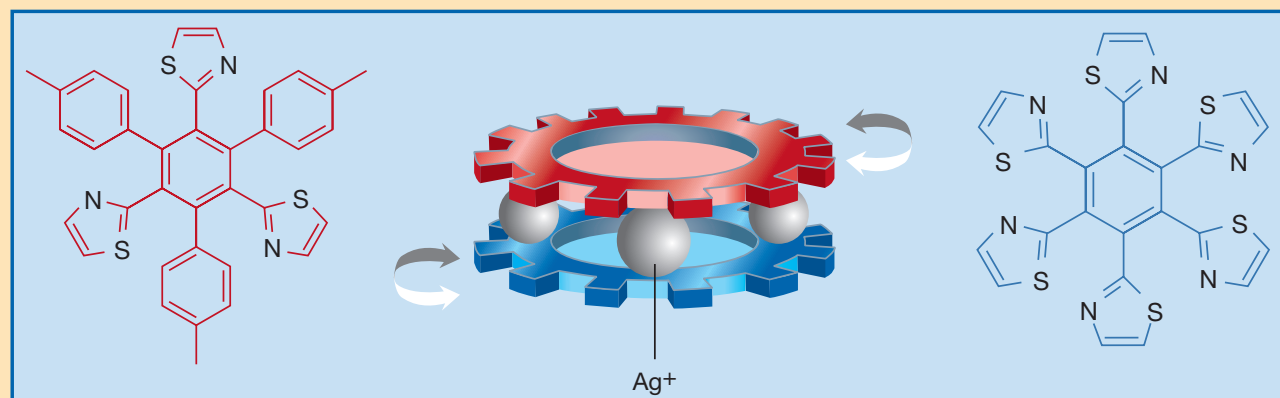

season to 25, 20 and 0 over the following years $^{2,3}$. Anecdotal reports began to circulate of local declines of white-backed vulture populations throughout India and also of a related species, the long-billed vulture Gyps indicus, which occurs in peninsular India and in southeast Pakistan. In consequence, a conference was convened in Mumbai in August 1999 by Asad Rahmani, director of the Bombay Natural History Society, to consider the possible causes. In the following year, numbers of the slender-billed vulture Gyps tenuirostris were also found to be declining rapidly over its range in northern and northeast India. East of the subcontinent, relict populations of white-backed and slenderbilled vultures survive in northern Cambodia, but very little is known of their status.

The meeting at Mumbai had a parallel with events 34 years earlier. Then, reports of the local disappearance of peregrine falcons, Falco peregrinus, prompted a conference that was held in Madison, Wisconsin, at the behest of J. J. Hickey ${ }^{4}$. Here, too, explanations for the population declines were proposed, but none could gain adequate support. At the Madison meeting, Derek Ratcliffe described his observations in Great Britain of peregrine falcons eating their own broken eggs ${ }^{4}$. In a now-classic paper ${ }^{5}$, published in 1967, he demonstrated that a physical change had begun in about 1947 in the form of reduced weights of the eggshells of peregrines and of several other species of raptorial bird. Only after an additional two years of intensive research could it be concluded that a new environmental contaminant in the form of the DDT-derived compound DDE (dichlorodiphenyldichloroethylene) was responsible for both the shell thinning of peregrine falcon eggs in Britain and the extinction of breeding peregrines in the eastern United States.

After a comparable time from the 1999 Mumbai conference, Oaks et al. ${ }^{1}$ demonstrate that the collapse of vulture populations in Pakistan has also been caused by a new environmental poison. Diclofenac is a very different kind of toxin from DDT, and travels along a very different pathway. But these findings are likely to have an equivalent impact to that produced by Ratcliffe's 1967 paper.

The Peregrine Fund, based in Boise, Idaho, began investigations of the vulture mortalities in Pakistan in 2000, in collaboration with the Ornithological Society of Pakistan. Parallel investigations were under way in India by the Bombay Natural History Society, in collaboration with the UK Royal Society for the Protection of Birds. Both groups initially focused on the search for a new disease factor, possibly one that had 'jumped' from another species; a disease hypothesis had remained the only plausible explanation for the observed mortalities ${ }^{6,7}$.

In both countries, necropsies of birds that had died suddenly in good physical condition showed that they had suffered from visceral gout - the accumulation of uric acid throughout the body cavity following kidney malfunction. Kidney failure has been an infrequent but serious side effect of the nonsteroidal anti-inflammatory drugs that have come into widespread use over the past 30 years $^{8-10}$. That the vulture mortalities might be caused by a veterinary medicine is not a hypothesis that comes easily from a wildlife biologist. But it emerged more naturally from two of the veterinarians working with the Peregrine Fund in Pakistan, Lindsay Oaks and Martin Gilbert, who put it to the test.

An initial survey indicated that diclofenac, introduced as a human medicine in the 1970 s as a painkiller and antiinflammatory agent, had recently come into widespread use in Pakistan as a veterinary medicine. Oaks et al. found that kidneys of vultures dying with the visceral gout syndrome contained residues of diclofenac, whereas vultures dying of other, known, causes did not. They also found that doses prescribed for domestic animals, and meat from domestic animals treated with such doses, were lethal to captive vultures, which died with the same symptoms as observed in wild birds. Oaks et al. are careful to limit their conclusions to Pakistan. But diclofenac is widely used as a veterinary medicine in India $^{11}$, where populations of all three species continue to decline; the conclusions apply also to Nepal and Bangladesh.

Has this discovery come in time to save the three species from extinction in the subcontinent? Banning most uses of DDT permitted the recovery of peregrine falcons, but adequate, although more expensive, 